\title{
New Family of Functionalized Monomers Based on Amines: A Novel Synthesis that Exploits the Nucleophilic Substitution Reaction
}

\author{
Lissette Agüero ${ }^{1}$, Luis G. Guerrero-Ramírez ${ }^{2}$, Issa Katime² \\ ${ }^{1}$ Grupo de Hidrogeles, Departamento de Química Macromolecular, Centro de Biomateriales, Universidad de La Habana. Ave. \\ Universidad s/n, La Habana, Cuba; ${ }^{2}$ Grupo de Nuevos Materialesy Espectroscopia Supramolecular, Departamento de Química-Física, \\ Facultad de Ciencia y Tecnología (Campus Leioa), Universidad del País Vasco, Bilbao, Spain. \\ Email: lissette@biomat.uh.cu, guillermo.guerrero@red.cucei.udg.mx, issa.katime@ehu.es
}

Received May $7^{\text {th }}, 2010$; revised April 17 $7^{\text {th }}, 2010$; accepted June $22^{\text {nd }}, 2010$.

\begin{abstract}
Chemistry modifications are usually performed to introduce specific group that can increase properties and functionality of materials. In this study, we present the synthesis of six new functionalized monomers prepared by nucleophilic substitution reactions. Reaction of aliphatic and aromatic amines with acryloyl chloride at $-20^{\circ} \mathrm{C}$, in presence of triethylamine allowed the synthesis of the corresponding amides. Proton nuclear magnetic resonance $\left({ }^{1} H\right.$ NMR $)$ spectroscopy, Fourier-transform infrared (FTIR) spectroscopy and ultraviolet- visible (UV-Vis) measurements confirmed the success of the synthesis with a yield over $90 \%$. These compounds emerged as potentially attractive monomers since they can be used to obtain stimuli-sensitive polymeric materials, due to the presence of amide and pyridine groups.
\end{abstract}

Keywords: Functional Monomers, FTIR, NMR, Polymer Design, UV-Vis

\section{Introduction}

One of the most powerful tools in the design of new polymers is the synthesis of new functional monomers capable of giving to the final polymer product tailor made properties [1-4]. For this purpose various kinds of synthesis strategies have been developed, e.g, protonization, complexation of metals, Dies-Alder reaction, bimolecular reaction, among others [5-7]. Nucleophilic substitution is a fundamental class of substitution reactions in which an "electron rich" nucleophile selectively bonds or attacks the positive or partially positive charge of an atom attached to a group or atom called as leaving group; the positive or partially positive atom is referred to as an electrophile [8-11]. For the last decades, the synthesis of monomers chemically structured has been focused mainly on nucleophilic substitution reactions, due to its uses easily allowing conversion simple and inexpensive compound into complex molecules [12-15]. Recent researches show that the synthesis of monomers for a specific syst$\mathrm{em}$, is carried out using complex synthetic procedures with a low yield [16-19]. Therefore the objective of this study was to synthesize with high efficiency six new monomers containing amides groups. The structures of the obtained functionalized monomers were characterized by ${ }^{1} \mathrm{H}$ NMR, FTIR and UV-Vis spectroscopy.

\section{Materials and Methods}

\subsection{Materials}

4-aminomethyl pyridine (4AMP) (Aldrich 98\%), 2-am inopyridine (2AP) (Merck 98\%), 2-diethylamino-ethylamine (2DEAEA) (Aldrich 98\%), ethylene diamine (ED) (Aldrich 98\%), 2-aminomethyl pyridine (2AMP) (Merck 98\%), 2-diisopropylamino ethylamine (2DIPAEA) (Fluka 99\%), acryloyl chloride (CA) (Aldrich 96\%), di-tertbutyl dicarbonate (BOC) (Aldrich 97\%), triethylamine (TEA) (Aldrich, 98\%), dichloromethane (DM) (Aldrich 98\%) and ethyl acetate (EA) (Panreac 99\%) were used as received without further purification.

\subsection{Characterization Techniques}

\subsubsection{FTIR Measurements}

Monomer's spectra were collected using Attenuated Total Reflectance (ATR) with a Smart Orbit accessory coupled with a Fourier Transform Infrared spectrophotometer FTIR (Nicolet 6700). All spectra were obtained from 
an average of 100 scans with $4 \mathrm{~cm}^{-1}$ of resolution.

\subsubsection{Nuclear Magnetic Resonance (NMR) Measurements}

The ${ }^{1} \mathrm{H}$ NMR spectra of the monomers were obtained using $\mathrm{D}_{2} \mathrm{O}$ as a solvent in a Bruker ACE instrument $(250$ $\mathrm{MHz})$ at $20^{\circ} \mathrm{C}$; chemical shifts $(\delta)$ were measured in ppm relative to deutered water $(\delta=7.26)$. Molecular structure of the monomers was determined using this technique.

\subsubsection{Ultraviolet-Visible Spectroscopy (UV-Vis) Measurements}

UV-Vis spectroscopy is a useful technique that helps to confirm the synthesis of the monomers because the amine-based monomers present an absorption band in this region of the electromagnetic spectrum. All the UV-Vis spectra were recorded using a CINTRA 303 spectrometer equipped with a power peltier supply thermocell control.

\subsection{Synthesis of Structured Monomers}

To obtain the functionalized monomers, the commercial monomers 4AMP, 2AMP, 2AP, 2DIPAEA and 2DEAEA were used and NHBOC monomer was synthesized in our laboratory using a method previously reported by Katime et al. [20]. The design of these monomers is targeted to provide $\mathrm{pH}$-sensitivity to the final products since the presence of ionizable groups can confer a selective behavior to local variations in $\mathrm{pH}$ values. Figure 2 shows the chemical structure of the $\mathrm{N}$-(pyridin-4-ylmethyl) acrylamide (NP4MAM), N-(pyridin-2-ylmethyl) acrylamide (NP2MAM), N-(pyridin-2-yl) acrylamide (NP2AM), N-(2-(diet- hyl amino)ethyl) acrylamide (2DEAEAM), N-(2-(diisopropylamino)ethyl) acrylamide (2DIPAEAM) and tert-butyl 2-acrylamidoethyl carbamate (2AAECM) amine-based monomers synthesized on this work. The synthetic procedure to obtain NP4MAM, NP2MAM, NP2AM, 2DEAEAM, 2DIAEAM and 2AAECM monomers is as follows according to the stoichiometry of the reaction (Figure 1), in a three mouths flask equipped with a condenser, addition funnel, and a temperature sensor, is placed the needed amount of acryloyl chloride previously dissolved in dichloromethane $(50 \mathrm{~mL})$. The reaction is carried out at a temperature of approximately $-20 \pm 5^{\circ} \mathrm{C}$ using liquid nitrogen and keeping the reaction mixture under constant magnetic stirring. In the addition funnel is introduced the precursor reagent (4AMP, 2AMP, 2AP, 2DEAEA, 2DIPAEA or NHBOC) previously dissolved in dichloromethane $(20 \mathrm{~mL})$. Additionally, to neutralize the hydrochloric acid that is formed as secondary product of the reaction triethylamine is added to the funnel. Once reached the reaction temperature, the mixture (reagent/TEA) is poured dropwise into the acryloyl chloride. In all cases a light colored viscous solution is obtained after the complete addition of the reagent/TEA mixture. Once the addition is completed, the dichloromethane is extracted by rotoevaporation at $35^{\circ} \mathrm{C}$ and the clear oil obtained is dissolved in distilled water (solution A). The monomer is extracted from the solution A by ethyl acetate using a separation funnel and then finally the monomer is obtained by rotoevaporation at $40^{\circ} \mathrm{C}$.

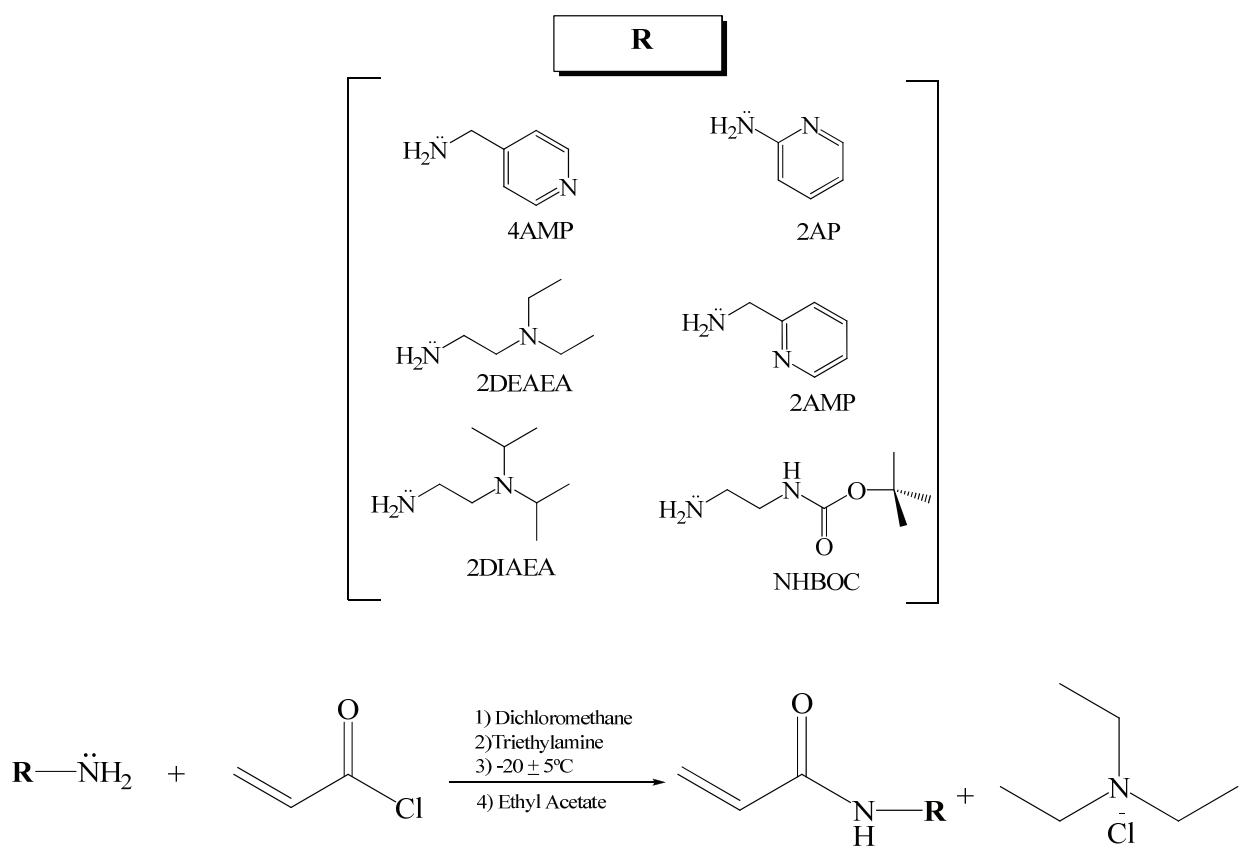

Figure 1. Stoichiometry of the synthesis reaction for obtain of structured monomers 
<smiles>C=CC(=O)NCc1ccncc1</smiles>

$N$-(pyridin-4-ylmethyl)acrylamide (NP4MAM)<smiles>C=CC(=O)NCCN(CC)CC</smiles>

$N$-(2-(diethylamino)ethyl)acrylamide (2DEAEAM)<smiles>C=CC(=O)NCCN(C(C)C)C(C)C</smiles>

$N$-(2-(diisopropylamino)ethyl)acrylamide 2DIPAEAM)<smiles>C=CC(=O)Nc1ccccn1</smiles>

$N$-(pyridin-2-yl)acrylamide (NP2AM)<smiles>C=CC(=O)NCc1ccccn1</smiles>

$N$-(pyridin-2-ylmethyl)acrylamide (NP2MAM)<smiles>C=CC(=O)NCCNC(=O)OC(C)(C)C</smiles>

tert-butyl 2-acrylamidoethylcarbamate (2AAECM)

Figure 2. Chemical structure of the amine-based monomers

\section{Results and Discussion}

\subsection{FTIR Studies}

Figure 3 shows the spectra of the precursor reagents (a) and the obtained monomers (b). Spectra (a) shows for the reagents that are pyridine-based (4AMP, 2AMP and 2AP) the most characteristic vibration bands of aromatic compounds, the vibration band due to stretch of the hydrogen of the aromatic ring from pyridine $\left(3060 \mathrm{~cm}^{-1}\right)$ and the band due to the vibration of the $\mathrm{CH}$ groups $\left(2920 \mathrm{~cm}^{-1}\right)$. Moreover, there are also confirmation signals in the region of $690-900 \mathrm{~cm}^{-1}$ due to bending vibration out of plane of the aromatic carbons. When the precursor reagents are amine-based the above mentioned vibrations bands disappear but other vibration bands can be appreciated. We can see several bands that remains, the most significant appear at 3100,1650 , and $1550 \mathrm{~cm}^{-1}$ and correspond to the amine group in all cases. The characteristics bands at 2950,13951375 and $1340 \mathrm{~cm}^{-1}$ correspond to the vibration of the isopropyl group from 2DIAEA, while absorption at $1180 \mathrm{~cm}^{-1}$ could be assigned to the vibration of ethyl group from 2DEAEA. On the other hand, the bands at 1140 and $1470 \mathrm{~cm}^{-1}$ are for the vibration of the tertbutoxy group from NHBOC. As can be seen in all cases, when the synthesis reaction with acryloyl chloride has been performed (spectra b) the signal of the carbonyl group appears in all the spectra corroborating the coupling of the acrylic group to the reagent structure, this ba- nd is due to the stretching vibration of the carbonyl group located at $1734 \mathrm{~cm}^{-1}$. In addition, it resolves a sig- nal at $1650 \mathrm{~cm}^{-1}$ which is due to stretch vibration of the double bond carbon-carbon for NP4MAM, NP2MAM and NP2AM monomers; signal that is coupled with sign- als own of the benzene ring that have a system of piconjugated double bonds and that is located at $1600 \mathrm{~cm}^{-1}$. Moreover, it is also appreciable the vibration of the $\mathrm{C}-\mathrm{H}$ group from a vinyl functional group located at $1210 \mathrm{~cm}^{-1}$ in all cases. The above results led us to the conclusion that the nucleophilic substitution reaction was carried out successfully in each case.

\section{2 ${ }^{1} \mathrm{H}$ NMR Studies}

Figure 4 shows the ${ }^{1} \mathrm{H}$ NMR spectra obtained for the NP4MAM (a), NP2MAM (b), NP2AP (c), 2DIPAEAM (d), 2DEAEAM (e) and 2AAECM (f). In all spectra can be appreciated the chemical shifts of the acrylic double bonds corresponding to the incorporation of the vinyl group from the acryloyl chloride (Table 1). Hence, these signals indicate that the vinyl group was coupled correctly in all cases because as noted in the spectrum (g) the acryloyl chloride presents displacements on its vinyl protons 5.9, 6.3 and $6.6 \mathrm{ppm}$, respectively. This change in the value of the chemical shifts is a clear indication that the chemical environment of these protons changed as a result of the coupling reaction. For spectra (a), (b) and (c), the signal from the aromatic ring from the pyridine 


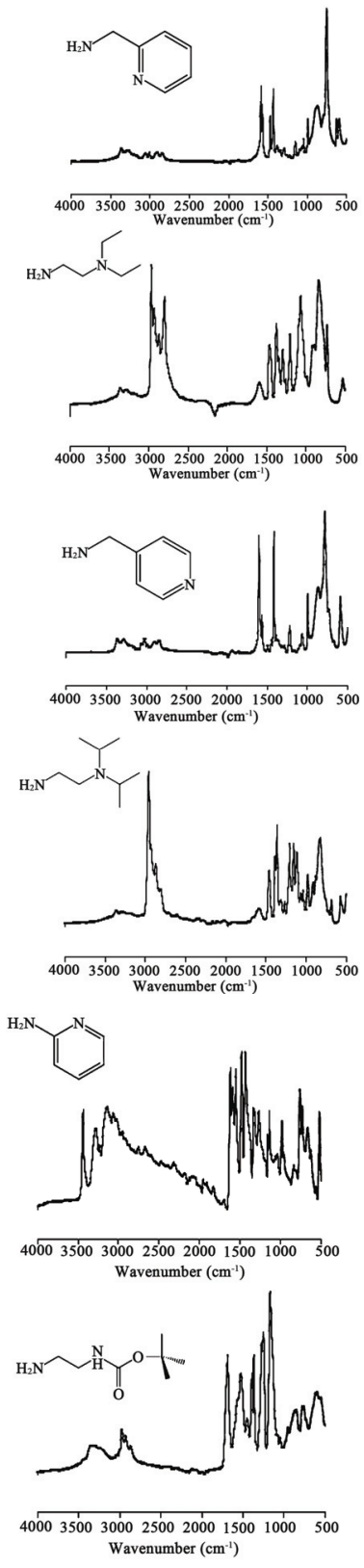

(a)

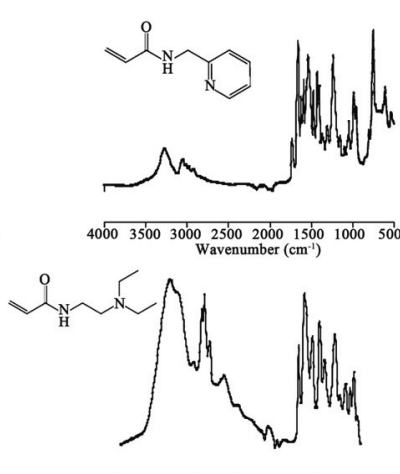

$4000 \quad 35003000250020001500 \quad 1000500$

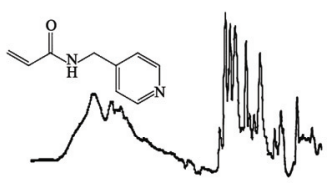

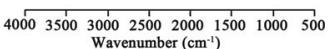

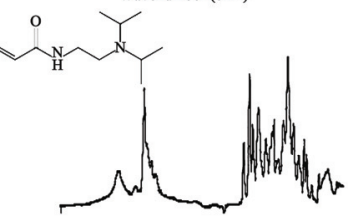

$4000 \quad 3500300025002000 \quad 1500 \quad 1000500$

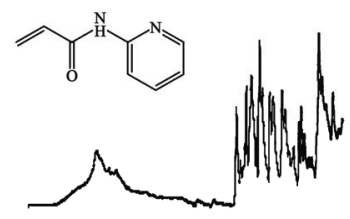

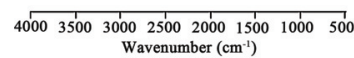

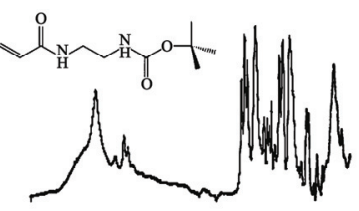

$4000 \quad 3500 \quad \begin{gathered}3000 \quad 2500 \quad 2000 \quad 1500 \quad 1000 \\ \text { Wavenumber }\left(\mathrm{cm}^{-1}\right)\end{gathered}$

(b)

Figure 3. FTIR spectra of (A) precursor reagents and (B) functionalized monomers synthesized by nucleophilic substitution reactions over the carbonylic carbon on the acryloyl chloride

group are located at 7.3 and $8.4 \mathrm{ppm}, 7.3,7.7$ and 8.2 ppm and 6.8, 7.5 and $8.1 \mathrm{ppm}$, respectively, these signals present values which are different of the precursors, in each case being 7.2 and $8.3 \mathrm{ppm}$ for 4AMP, 7.2, 7.6 and $8.3 \mathrm{ppm}$ for 2AMP and 6.7, 7.5 and $8.0 \mathrm{ppm}$ for 2AP. In spectrum (d) the signals located at 1.0 and $2.6 \mathrm{ppm}$ are produced by the isopropyl group from the precursor
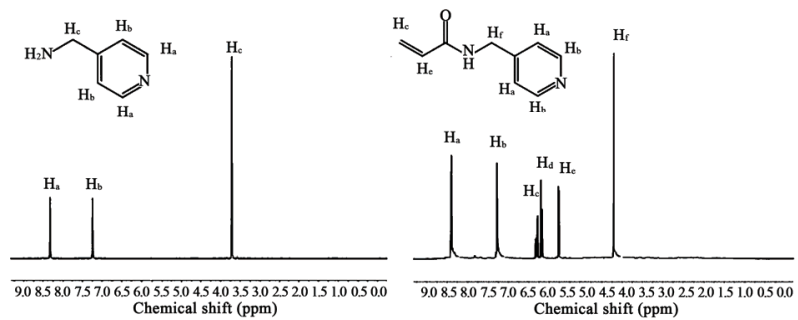

(a)
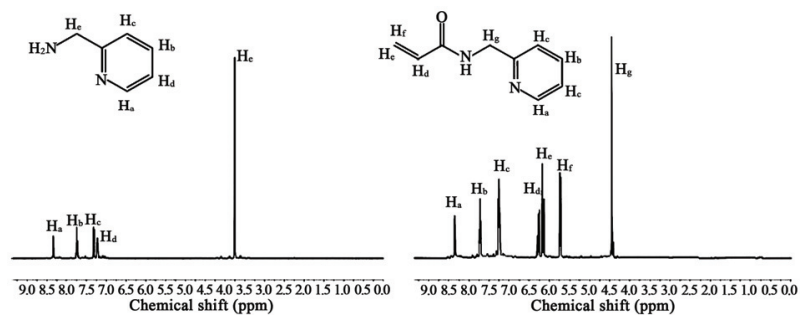

(b)

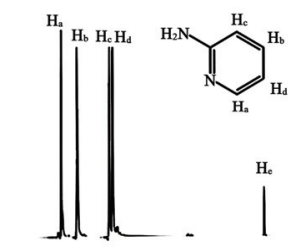

9.0858 .0757 .065605550454035302520151 .00500<smiles>CC(C)C(CCN)C(C)C</smiles>

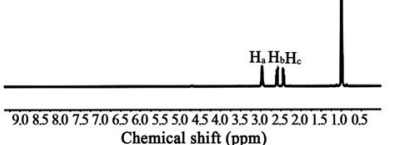

(d)
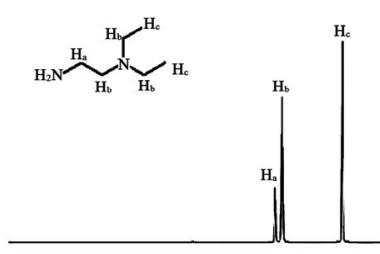

9.0858 .07 .57 .0656 .05550454 .0353 .0252 .0151 .00 .5 Chemical shift (ppm)

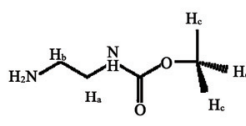

(e)
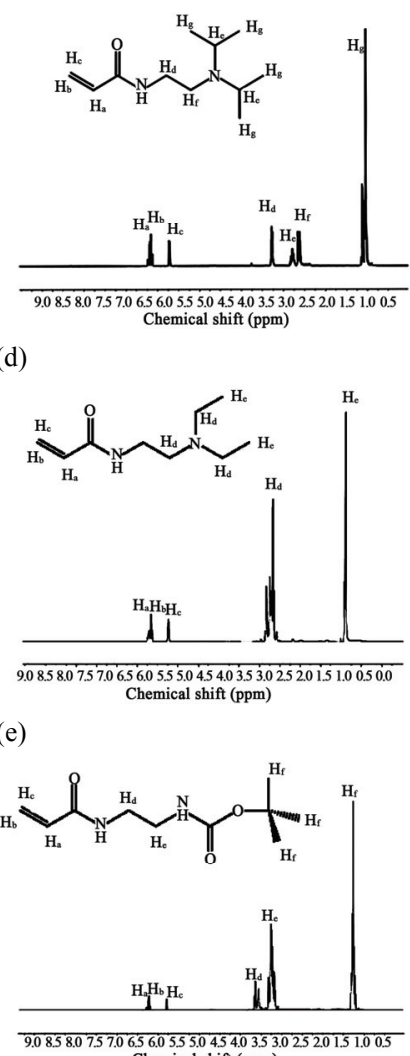

(f) 


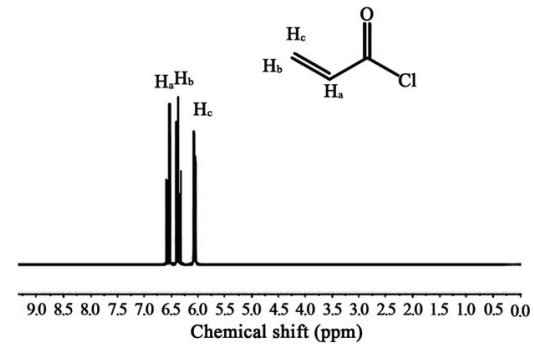

(g)

Figure 4. ${ }^{1} \mathrm{H}$ NMR spectra of NP4MAM (a), NP2MAM (b), NP2AP (c), 2DIPAEAM (d), 2DEAEAM (e), 2AAECM (f) and acryloyl chloride (g)

Table 1. Chemical shifts of the vinyl protons after the coupling reaction with acryloyl chloride

\begin{tabular}{cccc}
\hline Sample ID & $* \boldsymbol{\delta}_{\mathbf{a}}$ & $* \boldsymbol{\delta}_{\mathbf{b}}$ & $* \boldsymbol{\delta}_{\mathbf{c}}$ \\
\hline CA & 5.9 & 6.3 & 6.6 \\
NP4MAM & 5.7 & 6.2 & 6.3 \\
NP2MAM & 5.6 & 6.2 & 6.3 \\
NP2AM & 5.8 & 6.3 & 6.4 \\
2DIPAEAM & 5.6 & 6.2 & 6.3 \\
2DEAEAM & 5.6 & 6.2 & 6.3 \\
2AAECM & 5.7 & 6.2 & 6.3 \\
\hline
\end{tabular}

$* \delta_{\mathrm{a}}, \delta_{\mathrm{b}}$ and $\delta_{\mathrm{c}}$ are the chemical shifts of the vinyl protons.

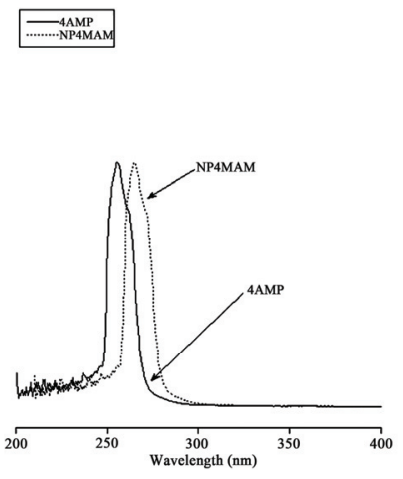

(a)

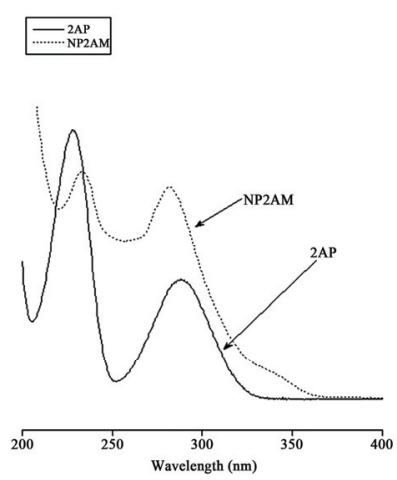

(c)

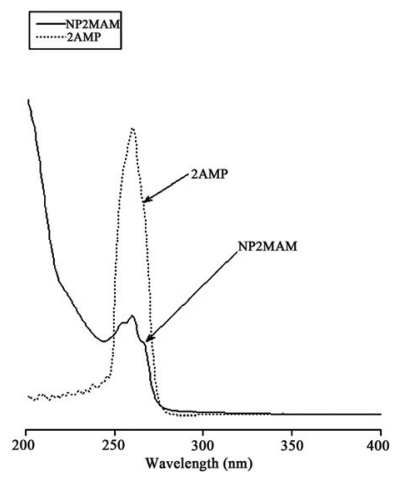

(b)

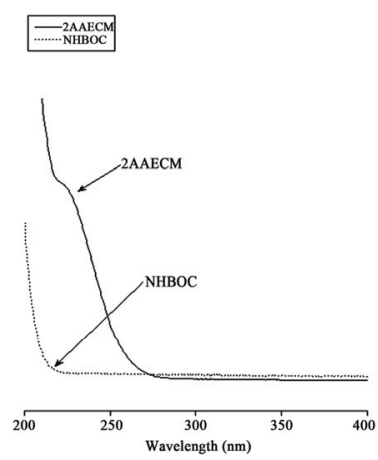

(d)

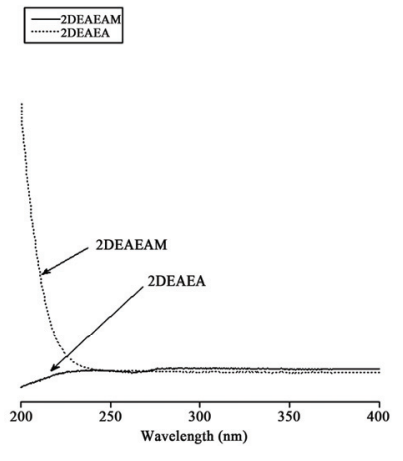

(e)

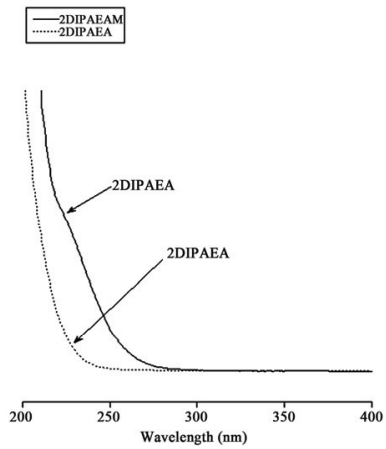

(f)

Figure 5. UV-Vis spectra of precursor reagents and pre-functionalized monomers. (a) 4AMP-NP4MAM; (b) 2AMPNP2MAM; (c) 2AP-NP2AP; (d) 2DIPAEA-2DIPAEAM; (e) 2DEAEA-2DEAEAM; (f) NHBOC-2AAECM

2DIPAEA. In the case of the 2DEAEAM monomer (e) the signals at 1.1 and $2.4 \mathrm{ppm}$ are due to the ethyl group and finally, in spectrum (f) the chemical shift of 1.3 corresponds to the tert-butoxy group from the NHBOC precursor. ${ }^{1} \mathrm{H}$ NMR characterization also confirm the chemical structure of amides obtained.

\subsection{UV-Vis Studies}

As can be seen in Figure 5, in all UV-Vis spectra there are differences between the precursor reagent and the products of the nucleophilic reaction. In the case of NP4MAM monomer (a), there is a displacement of the maximum absorption wavelength from 261 to $274 \mathrm{~nm}$, due to the vinyl group incorporated for NP2MAM (b) there is a bathochromic shift due to the decrease of the molar absorption coefficient that is caused by the double bond from the vinyl group, but in spectrum (c) appears a batochromic shift from 230 to $240 \mathrm{~nm}$ and a hypsochromic shift from 293 to $286 \mathrm{~nm}$, this may be due to the structure of the molecule as well as to the incorporation of the vinyl group because the 2AP do not present the methyl group as is the case of 4AMP and 2AMP. For the monomers 2DIPAEAM (d), and 2DEAEAM (e), there is not significant changes on the spectra because the precursor reagents of each case do not have an aromatic ring that causes UV-Vis absorption but in spectrum of 2AAECM (f) exists a weak absorption band near to $215 \mathrm{~nm}$ product of the coupling reaction.

\section{Conclusions}

The synthesis of chemically structured monomers by nucleophilic substitution reactions is a simple way to obtain molecules capable to be used as a potential precursors in the design of new synthetic devices by radical polymerization that may be used in different areas of the chemical science such is the case in biomedicine acting as a drug 
carriers. The analysis by UV-Vis spectroscopy, FTIR spectroscopy and NMR spectroscopy confirms the success of the coupling reaction with acryloyl chloride.

\section{Acknowledgements}

Financial support for this work was provided by Ministerio de Ciencia e Innovación (Project Number: EUI200800178) and the scholarships from Ministerio de Asuntos Exteriores y Cooperación, Spain (MAEC-AECID) to whom researches are gratefully acknowledged.

\section{REFERENCES}

[1] L. Pérez, V. Sáez, E. Hernaz, E. Rodríguez and I. Katime, "Synthesis and Characterization of Reactive Copolymeric Microgels," Polymer International, Vol. 54, No. 6, 2005, pp. 963-971.

[2] L. G. Guerrero-Ramírez, S. M. Nuño-Donlucas, L. C. Cesteros and I. Katime, "Smart Copolymeric Nanohydrogels: Synthesis, Characterization snd Properties," Material Chemistry and Physics, Vol. 112, No. 3, 2008, pp. 10881092.

[3] L. G. Guerrero-Ramírez, S. Nuño-Donlucas, L. C. Cesteros and I. Katime, "Novel Functionalized Nanohydrogels, Synthesis and some Applications," Journal of Physics Conference Series, Vol. 127, No. 1, 2008, pp. 1-10.

[4] R. Arshady, "Microspheres for Biomedical Applications: Preparation of Reactive and Labeled Microspheres," Biomaterials, Vol. 14, No. 1, 1993, 5-15.

[5] J. P. Clayden, N. Greeves, S. G. Warren and P. D. Wothers, "Organic Chemistry," 1st Edition, Publishing Ltd., Oxford, 2000.

[6] J. J. Shiers, M. Shipman, J. H. Hayes and A. M. Z. Slawin, "Rare Example of Nucleophilic Substitution at Vinylic Carbon with Inversion: Mechanism of Methyleneaziridine Formation by Sodium Amide Induced Ring Closure Revisited," Journal of American Chemical Society, Vol. 126, No. 22, 2004, pp. 6868-6869.

[7] S. Thamizharasi, P. Gnanasundaram and S. Balasubramanian, "Synthesis, Characterization and Reactivity Ratios of Copolymers Derived from 4-Nitrophenyl Acrylate and N-Butyl Methacrylate," Journal of Applied Polymer Science, Vol. 88, No. 7, 2003, pp. 1817-1824.

[8] J. M. J. Frechet and L. J. Nuyens, "Use of Polymers as Protecting Groups in Organic Synthesis. III. Selective
Functionalization of Polyhydroxy Alcohols," Canadian Journal of Chemistry, Vol. 54, No. 6, 1976, pp. 926-934.

[9] A. Dasgupta and S. Sivaram, "Polymerization of Monomers Bearing Amide Functionalities," Macromolecules, Vol. 27, No. 1, 1994, p. 165.

[10] A. P. Krapcho and C. S. Kuell, "Mono-(BOC)-Protected Diamines. Synthesis of Tert-Butyl-N-Alkyl-N-(2-Aminoethyl) Carbamates and Tert-Butyl-N-[2-(Alkylamino) Ethyl] Carbamates," Synthetic Communications, Vol. 23, No. 17, 1993, pp. 2443-2449.

[11] L. Pérez, V. Sáez, E. Hernaz and I. Katime, "Novel pH and Temperature Responsive Methacrylamide Microgels," Macromolecular Chemistry and Physics, Vol. 210, No. 6, 2009, pp. 467-477.

[12] P. H. Toy and K. D. Janda, "Soluble Polymer-Supported Organic Synthesis," Chemical Research, Vol. 33, No. 8, 2000, pp. 546-554.

[13] D. C. Snyder, "Conversion of Alcohols to Chlorides by TMSCL and DMSO," Journal of Organic Chemistry, Vol. 60, No. 8, 1995, pp. 2638-2639.

[14] J. March, "Advanced Organic Chemistry," 4th Edition, Wiley, New York, 1992.

[15] W. C. Matthew and P. H. Toy, "An Improved and General Synthesis of Monomers for Incorporating Trityl Linker Groups into Polystyrene Synthesis Supports," Tetrahedron, Vol. 60, No. 12, 2004, pp. 2903-2907.

[16] A. Gomtsyan, "Direct Synthesis of $\beta$-Aminoketones from Amides via Novel Sequential Nucleophilic Substitution/Michael Reaction," Organic Letters, Vol. 2, No. 1, 2000, pp. 11-13.

[17] R. A. Rossi and R.H. de Rossi, "Aromatic Substitution by the SRN1 Mechanism," ACS Monograph Series, American Chemical Society, No. 178, 1983, p. 178.

[18] N. S. Imyanitov, "Electrophilic Bimolecular Substitution as an Alternative to Nucleophilic Monomolecular Substitution in Inorganic and Organic Chemistry," Journal of General Chemistry, Vol. 60, No. 3, 1990, pp. 417-419.

[19] J. Sudimack and R. Lee, "Targeted Drug Delivery via the Folate Receptor," Advanced Drug Delivery Reviews, Vol. 41, No. 2, 2000, pp. 147-162.

[20] V. Saéz, L. Pérez, M. T. Herrero, E. Hernaz and I. Katime, "Chemical Functionalization of Poly (Nitrophenyl Acrylate-Co-Methacryl-Amide) Microgels with Pyridines to Provide them Specific PH-Sensitivity," Polymer, Vol. 47, No. 2, 2006, pp. 818-819. 